\title{
Enduring influence of the purpose of experiences: Encoding-retrieval interactions in word and pseudoword perception
}

\author{
BRUCE W. A. WHITTLESEA and ALISA L. CANTWELL \\ Mount Allison University, Sackville, New Brunswick, Canada
}

\begin{abstract}
Letters in words are identified more easily than are letters in nonwords or letters alone. These effects may depend on separate representations of general lexical attributes and of specific contexts, or on memory for particular experiences. We required subjects to associate meanings with some pseudowords and to perform a physical analysis on others. After $24 \mathrm{~h}$, subjects identified pseudowords associated with meanings more accurately than novel or physically analyzed pseudowords. However, perceptual accuracy was independent of recall of meanings, suggesting that meaning was not available as a context-free resource. Instead, perceptual accuracy was correlated with the interdependence of stimulus components in perception, suggesting that encountering pseudowords for different purposes had caused different perceptual organizations, which exercised lasting influence. We concluded that the perceptual advantage of words may be incidental to the purposes for which words are ordinarily processed, and may depend on preservation of particular perceptual experiences.
\end{abstract}

Letters in words are generally easier to identify than the same letters presented either in nonwords or alone (Reicher, 1969; Wheeler, 1970). Although this advantage clearly depends on the perceiver's experience of words, it is less clear what information about words is retained in memory or how that information guides subsequent perception. Early investigations demonstrated that letters in orthographically regular pseudowords could be identified as easily as letters in natural words (Baron \& Thurston, 1973), suggesting that the advantage was not due to the meaning or the specific familiarity of words. Instead, perceptibility was found to be correlated with a variety of aspects of stimulus regularity, including word frequency (Wheeler, 1970), sequential dependency (Rumelhart \& Siple, 1974), positional frequency (Mason, 1975), cluster frequency (Estes, 1975), and pronounceability (Gibson, Shurcliff, \& Yonas, 1970). These observations suggested that perception is guided by memory for abstract properties, such as the structural units of which words are composed (Estes, 1975) or the structural interrelations among whole words (McClelland \& Rumelhart, 1981).

These observations were gathered in experiments comparing the perceptibility of items differing in selected lexical properties. In contrast to this cross-sectional approach, some investigators have adopted a longitudinal approach, examining the effects of earlier experiences of an item on its subsequent perceptibility. Evidence from studies of this type suggests that the specific familiarity of an item

Preparation of this article was supported by NSERC Grant A0573. We wish to acknowledge with gratitude the editorial advice of Lee Brooks, Larry Jacoby, and Henry James in the preparation of this article. Requests for reprints should be sent to Bruce Whittlesea, Department of Psychology, Mount Allison University, Sackville, NB E0A 3C0, Canada. may have a greater impact on perception than previous work suggested. For example, items are identified more easily in later than in earlier presentations, and the effects of an earlier presentation may be strong and enduring (Jacoby, 1983a; Jacoby \& Dallas, 1981; Kolers, 1976). Moreover, the facilitation of subsequent exposures appears to depend not simply on repetition of the item itself, but also on reinstatement of contextual elements such as symbolic domain (Warren \& Morton, 1982), list context (Jacoby, 1983a), and typeface (Kolers, Palef, \& Stelmach, 1980). Thus perception appears to depend on the episodic detail of previous experiences as well as on stimulus regularity.

Two alternative explanations are available to integrate these findings. First, memory may maintain separate stores of information about particular events and general regularities (e.g., Cohen \& Squire, 1980; Tulving, 1972). In this interpretation, a conceptual or semantic memory system retains information about general lexical properties such as the frequency of words (Morton, 1979) or letter clusters (Estes, 1975), and an episodic system preserves information about the contexts in which items were encountered on particular occasions (Tulving, 1983). These systems are separately responsible for observations of perceptual dependence on regularity and reinstatement of context. A second explanation is that memory maintains a code of each particular event experienced, complete in whatever detail was attended (e.g., Jacoby \& Brooks, 1984; Jacoby \& Witherspoon, 1982; Whittlesea, 1987). In this case, memory does not distinguish between the item and its context; instead, the context influences the experience of the item, and the experience is preserved. Dependence on regularity of the stimulus arises not because regularity information is directly coded, but 
because regular stimuli are similar to more events that the person has experienced.

We attempted to investigate whether the experience of an item in a context results in distinct information about item and context, or whether the experience is retained as a whole. Briefly, by manipulating the purpose for which subjects encountered stimuli, we observed that different purposes caused enduring differences in the accuracy of identification. These differences were apparently mediated by different organizations of the components of items, rather than by differences in structural or familiarity information independently. In consequence, we concluded that the memory supporting perception is best conceptualized as an episodic system preserving the subject's experiences of items.

\section{Meaning and Perception}

We elected to study the relationship between meaning and perception as a convenient test of the representations supporting perception. Although, as indicated previously, Baron and Thurston (1973) demonstrated that meaningfulness is not critical to perceptual superiority, meanings may assist perception if available. For example, meanings activated in an early stage of perception may constrain the sequence or depth of further perceptual processing. Alternatively, meaning may affect perception indirectly, as an encoding demand incidentally involving a perceptual experience. Because meaning belongs to the whole item, processing for meaning may occasion perceptual integration of the letters of the item, at least relative to other task demands. Integration of the elements could lead to a perceptual advantage in a later exposure, because identification of a particular component could be assisted by information about other components.

Thus meaning may influence perception either as an abstract property of the item or as the occasion for experiencing items in a particular way. These alternatives reflect the alternative accounts of memory discussed earlier. A direct influence of meaning on perception would be consistent with a dual-memory account that suggests that abstract properties may affect perception independent of the characteristics of particular experiences. However, if the effect of meaning is indirect, mediated by interdependent organization of the stimulus components in memory, then an episodic memory system that retains the experiences of particular events may offer a better account.

\section{EXPERIMENT 1}

To determine whether meaning has any independent effect on perception, other abstract properties such as frequency and orthographic regularity must be held constant while meaning is varied. Comparing the perceptibility of natural words and pseudowords selected to be comparable based on other characteristics achieves only an approximation of the desired control. We attempted to achieve this control directly, by manipulating the meaningfulness of pseudowords and comparing the perceptibility of meaningless items to the perceptibility of the same items when they were associated with meanings.

\section{Method}

Subjects. Thirty undergraduate students participated for course credit.

Apparatus. The experiment was conducted on an Apple IIe microcomputer using a Zenith monitor.

Procedure. We assembled a set of 24 orthographically legal, pronounceable CVCVC nonwords, selected to be maximally discriminable (no two letters co-occurred in the same positions in more than one item). Twelve items selected at random were assigned meanings (meaning condition), which the subjects were required to learn prior to a perceptual test. The remaining 12 items were not exposed before the test (novel condition). Assignment of items to conditions was counterbalanced between subjects, so that over subjects the items within conditions did not differ in preexperimental characteristics, such as pronounceability, orthographic regularity, frequency of letter clusters, or frequency of letters in particular positions.

The meanings consisted of easily understood concepts for which no familiar English word exists. Thus, for example, the subjects learned that a RAVIT is "a group of butterflies" and a WALEN is "the noise a dam makes before it breaks." Meaning items were exposed five times each in an expanding series, so that successive presentations occurred approximately $1,3,6$, and 10 trials after the first presentation. On the first presentation, the subject was shown the item together with its definition. The second, third, and fourth presentations required the subject to answer a question involving the item's meaning ("If you lived five miles below a dam, would you be worried about a WALEN?"), responding "yes" or "no" and providing an explanation of the answer. On the last presentation, the subject was asked to recall the meaning of the item. The correct definition of the item was presented on the monitor after each of these trials, providing the subjects an opportunity to correct themselves. These training trials were initiated and paced by the subject. These circumstances were designed to bring about nearperfect learning of associated meanings.

Directly after this task, we tested the subjects' ability to identify single letters in brief presentations of stimuli. Four types of stimuli were presented in random series, including the meaning and novel items, 12 moderately high-frequency CVCVC natural words, and 12 single letters. Before each trial, a fixation stimulus was presented, which consisted of arrows flanking the space in which an item would appear. Subjects initiated trials by striking a key, which caused a stimulus to be exposed for approximately $20 \mathrm{msec}$. Single letters were presented in the same screen location they occupied in one of the pseudowords, and the other four spaces were filled with pattern masks (rectangles filled with asterisks). In all cases, stimulus exposure was terminated by the presentation of a pattern mask in each of the five locations where a letter could occur. Simultaneously, a caret was presented under one of the five locations.

Immediately following each trial, subjects were required to report the identity of the letter that had been briefly presented in the location specified by the caret, guessing if necessary. The five positions were interrogated with approximately equal frequency within each stimulus type, and with identical frequency between stimulus types. In the case of single letters, the caret was always located where the letter had occurred.

\section{Results and Discussion}

The probability of identifying letters correctly in each condition of the perceptual task was: words, .69; meaning items, .62; novel items, .48; and single letters, .38. Analysis of variance indicated an overall effect of test con- 
dition $[F(3,87)=19.204, M S \mathrm{e}=.030, p<.001]$, and Newman-Keuls analysis indicated that all pairwise comparisons differed significantly $(p<.05)$, except the comparison of words and meaning items. Consistent with the findings of many previous studies of the word advantage (e.g., Reicher, 1969), these results demonstrate that a letter in a word is better perceived than a letter presented either alone or in a (novel) pseudoword.

The meaning pseudowords also exhibited perceptual superiority over both single letters and novel pseudowords. Critically, the difference between meaning and novel conditions cannot be accounted for in terms of any preexperimental properties of the items, such as frequency or orthographic regularity, because assignment of pseudowords to conditions was counterbalanced. It thus appears that the task of associating meanings with pseudowords has some independent effect on perception and results in a perceptual advantage analogous to the word advantage.

However, it cannot be concluded that the advantage is due to meaning per se. Repeated pseudowords may exhibit a perceptual advantage over novel pseudowords without benefit of meaning, and the size of the effect may rival that of the word advantage (Salasoo, Shiffrin, \& Feustal, 1985). Thus the meaning advantage may be due to exposure of these items prior to the perception test rather than to any aspect of meaning.

\section{EXPERIMENT 2}

If the effect of associating items with meanings is as nonspecific as this argument suggests, then preexposure of items in other tasks should cause equal perceptual facilitation. In Experiment 2 we exposed two sets of items to each subject prior to the perceptual test, one in the meaning task as before and the other in a letter-comparison task that did not involve meanings. As in Experiment 1, the items used in these two conditions were counterbalanced between subjects, thereby holding constant all item-level properties.

\footnotetext{
Method

Subjects. Thirty undergraduate students participated for course credit.

Procedure. In a training phase, all subjects received the 24 pseudowords used in Experiment 1. Twelve items were exposed in the meaning condition, as before. The remaining 12 items were shown in a letter-comparison task (compare condition), which required the subject to compare each letter of the stimulus with those of another letter string. For this task, the subjects were provided with a protocol on which two columns of five blank spaces appeared, the columns labeled "Same" and "Different." Each pseudoword (c.g., WALEN) was presented on the monitor with a comparison item (e.g., WELEP) appearing directly bencath it. The vertical space between the two stimuli was approximately half the height of a letter. The subject was required to copy each letter of the target pseudoword into the corresponding space on the protocol, but using the spaces of the "Same" and "Different" columns depending on whether the letter matched the corresponding letter of the comparison item. As in the meaning condition, each target item was presented live times, with the order of presentation yoked to the
}

order used in the meaning condition. In an attempt to match the variability of experience of meaning items afforded by the different questions, target items in the compare condition were paired with a different comparison item on each presentation, so that although the subjects copied the same letters in the same positions on each presentation of the stimulus, the assignment of a letter to "Same" or "Different" columns changed between trials. The items employed in the meaning and compare conditions were counter balanced between subjects, as was the order of the conditions.

The perceptual test was similar to that in Experiment 1, except that stimuli were exposed for $30 \mathrm{msec}$ before being terminated by the mask.

\section{Results and Discussion}

The probability of correct report in each condition was: words, .83; meaning, .83; compare, .68; and single letters, .58. Analysis of variance indicated an overall effect of test condition $[F(3,87)=20.745, M S e=.036$, $p<.001]$. Newman-Keuls a posteriori analysis revealed that all differences were significant $(p<.05)$, except the comparison of words and meaning pseudowords, as in Experiment 1 . Because both meaning and compare items were exposed before the test, the meaning advantage indicates that associating pseudowords with meanings has some effect on perception beyond simply granting an opportunity to see the item.

It is less clear whether the meaning advantage is due to a direct or indirect influence of meaning, to use of the meaning of the item in the act of perception or to use of previous experiences whose perceptual characteristics were determined by the demand to derive meaning. In either case, it is tempting to speculate that a similar mechanism underlies word perception, because the perceptibility of natural words is similar to that of meaning pseudowords and because both possess meanings. However, dual-memory accounts imply separate mechanisms for the two, because the words are experimentally novel, so that their perception should be assisted only by a semantic code, whereas the pseudowords, being re-presented in a familiar context, should be facilitated by an episodic code.

\section{EXPERIMENT 3}

One of the major arguments for a distinction between memory for general and memory for specific properties is provided by the observation of a dissociation between perceiving and remembering words (Cohen \& Squire, 1980; Jacoby \& Dallas, 1981; Jacoby \& Witherspoon, 1982; Tulving, Schacter, \& Stark, 1982). Coupled with the apparent stability of word perception across time and context (e.g., Morton, 1979) and the context-specific nature of remembering (e.g., Smith, 1979), the dissociation suggests that the two functions are served by independent memory systems, one retaining general, context-free aspects of experience, and the other preserving episodic detail (Tulving, 1983).

As indicated above, observations of enduring facilitation of perception following earlier presentations of an 
item require that episodic information has at least some role in word perception, and limit the identification of particular memory systems with particular tasks. However, the greatest challenge to the dual-memory hypothesis comes from observations that recognition and perception are affected in similar ways by similar variables. For example, Jacoby and Witherspoon (1982) demonstrated that performance on both tasks is specific to the contexts in which items were previously encountered, and Jacoby (1983b) showed that manipulation of the perceptual aspects of the encoding task affects word perception just as manipulation of nonperceptual aspects affects recognition.

These findings suggest that the apparent stability of word perception arises because of low variability of perceptual demands in encounters with words, rather than because perception relies on an inherently stable representation system. Such observations have led some authors to argue that the semantic-episodic distinction does not adequately capture differences in the use of memory, and to investigate the feasibility of a single memory retaining episodic detail about particular experiences (e.g., Brooks, 1987; Jacoby \& Brooks, 1984; Jacoby \& Witherspoon, 1982). This interpretation emphasizes task-dependent differences in stimulus processing, resulting in variations in the representation of an event, and task-dependent differences in accessing those representations resulting in dissociations among retrieval tasks.

One important aspect of stimulus processing that may vary with task demands is perceptual integration. Whittlesea (1987) observed that requiring subjects to copy words resulted in greater perceptual accuracy in a later test than did requiring letter-at-a-time comparison (as in the compare task used in Experiment 2 of this study). These findings were interpreted to suggest that the tasks encouraged different degrees of perceptual integration of stimulus components. The copying task was thought to cause relatively integral processing of components, because it encouraged treating them simultaneously as parts of a whole item, whereas the comparison task, which required attention to separate components, was thought to occasion relatively nonintegral processing. Greater perceptual integration of copied items would explain their greater perceptibility, because integration implies that perception of each component assists and is assisted by perception of other components through mutual generation. In support of this interpretation, perceptual interdependence of components (the probability of identifying a component given that another was identified) was found to be greater for copied items than for compared items.

This integration hypothesis may explain the perceptual advantage of meaning pseudowords over compare items in Experiment 2. Processing for meaning may incidentally involve greater perceptual integration than does the compare task, perhaps because it encourages treating the stimulus as a meaning-bearing unit, whereas the compare task focuses attention on the component letters individually. Greater perceptual integration would result in better perception, through the mutual dependence of the components.

This account suggests that the perceptual advantage of natural words is also due to preservation of episodic influence. Because the purpose of encountering words normally entails processing for meaning, most experiences of words would be expected to involve relatively integral processing, resulting in perceptual interdependence of the letters of words. Thus meaning may be indirectly responsible for the perceptual superiority of words, by serving as the occasion for integral perceptual operations.

However, the perceptual advantage of meaning over compare pseudowords may be due instead to maintenance of abstract, context-free information, as suggested by a dual-memory account. As discussed above, the meanings of the items may be directly involved in the act of perception, serving to constrain the possible identities of tested items. Alternatively, the attempt to associate meanings with pseudowords may cause the subject to attend more diligently, resulting in better learning of the item's structure. This hypothesis suggests that the strength of the trace depends on the task in which the item was encountered, but that the representation retains information about the structure of the item in canonical form. Experiment 3 was designed to compare these explanations with the episodic integration hypothesis.

\section{Method}

Subjects. Thirty undergraduate students participated for course credit.

Procedure. The subjects in Experiment 3 received the same meaning and compare training as did subjects in Experiment 2. Five types of items were exposed in the perceptual test, including novel pseudowords as well as the words, meaning and compare pseudowords, and single letters used in Experiment 2. In order to accommodate the extra pseudoword condition, the item pool was increased by 12 items. The resulting pool consisted of 36 orthographically legal, pronounceable CVCVC items in which no pair of letters cooccurred in the same positions in more than one item. Items from this pool were rotated through the three pseudoword conditions between subjects, occurring equally often in each condition.

Three additional changes were made to the procedure of earlier experiments. First, the subjects were required to report entire stimuli in the perception test, guessing if necessary, rather than a single letter as in Experiments 1 and 2 . This innovation permitted investigation of the interdependence of perceptual processing as well as the accuracy of perception, as discussed below. As in Experiment 1, stimulus presentation was terminated by a pattern mask after $20 \mathrm{msec}$. Protocols were scored for the number of letters reported in the correct spatial location. An incorrect letter in a particular location could result either from rearrangement due to positional uncertainty or from simple incorrect reporting. Although these errors may stem from different causes, both were treated as perceptual failure. Second, in order to assess the durability of perceptual facilitation afforded by experience of items in various tasks, the perceptual test was delayed until $24 \mathrm{~h}$ after exposure of meaning and compare items. Third, to determine whether the perceptual advantage of meaning items was due to their meanings per se, we tested 
the subjects' ability to recall meanings after the perceptual test. In this test, the meaning items were presented on a typed sheet, and the subjects were asked to state the meaning associated with each. Recall of a correct definition for the wrong item was counted as an error.

\section{Results and Discussion}

The probabilities of correctly reporting a letter in the perceptual task were: words, .75 ; meaning, .75 ; compare, .63; novel, .58; and single letters, .49. Analysis of variance indicated a significant effect of test condition $[F(4,116)=27.69, M S e=.014, p<.001]$, and Newman-Keuls a posteriori analysis indicated that all paired comparisons were significantly different $(p<$ .01 ), except the comparison of words and meaning items (as in Experiments 1 and 2).

The perceptual superiority of meaning over compare items and of compare over novel items occurred in spite of the fact that the items were rotated through all three conditions, so the differences could not be due to preexperimental properties. The meaning-compare difference also could not be due to lack of a memory code for compare items, since they were exposed, and the benefits of that exposure are reflected in the advantage of compare over novel items. Instead, the meaning-compare difference must be due to the difference in purpose for which items were encountered in the two conditions. The fact that this difference was measured $24 \mathrm{~h}$ after exposure to the items indicates enduring effects of task demands on perception.

As discussed earlier, the meaning-compare difference may occur because the meaning task encourage greater perceptual integration, or because the meaning task creates stronger traces, or because the meanings of meaning items are directly involved in the activity of perception. If perception automatically activates meanings, which in turn mediate perception, then perceptual accuracy should be correlated with success in identifying the meanings of meaning items. Similarly, if the effort involved in associating meanings with pseudowords results in strong traces, then greater effort should result in both stronger traces and stronger associations, again leading to a correlation. However, if traces differ in organization rather than strength, and if perception is primarily affected by differences in perceptual information, then no correlation between the tasks would be expected.

The correlation between the number of letters correctly identified in a meaning item and the probability of identifying its meaning was approximately zero (point-biserial $\left.r^{2}=.004\right)$ and nonsignificant $[t(358)=1.22]$. This low correlation was not caused by lack of variance in the perception data ( $S D=.19$ ) or floor or ceiling effects in the recall data (mean proportion recalled $=.54$ ). Equally, it was not due to subjects having learned incorrect definitions in the training phase, because accuracy in responding to questions about the meanings of items in that phase was near ceiling $(97.22 \%$ accuracy).

Instead, the independence of perception and meaning retrieval suggests that meaning affects pseudoword per- ception indirectly, ${ }^{1}$ by affecting the perceptual experience of the item and thus determining the information available to support later perception. To investigate the hypothesis that the important property of this experience is perceptual integration, we examined the relationship between the accuracy and interdependence of identifying stimulus components. We indexed the perceptual interdependence of components by means of phi, the Pearson correlation coefficient for four-fold tables (Fienberg, 1977). Because comparison of phi coefficients may slightly underrate differences in bivariate association due to differences in the marginal proportions between conditions, we also determined an estimate of the consistency of success in identifying various components of a stimulus.

The computation of these indices can be illustrated through consideration of the novel pseudoword condition. In this condition, each subject was tested on 12 items, each consisting of five letters. Considering only the first two letters of each item, four different outcomes may be distinguished: a hit on the first letter and a hit on the second, a hit and a miss, a miss and a hit, and a miss and a miss. These outcomes were evaluated for each of the 12 items for each of the 30 subjects, resulting in 360 pairs of guesses distributed across a four-fold table. Chi-square was computed from the marginal values of the table, and converted to phi $\left.\left[\Phi=\chi^{2} / N\right)^{1 / 2}\right]$, and the probability of consistent outcomes was computed by adding the hit-hit and miss-miss probabilities.

The five positions in which letters occurred can be selected in 10 pairs, and the two indices were computed for each pair. The entire operation was then repeated for the word, meaning, and compare conditions, resulting in two sets of 10 estimates of bivariate dependence for each condition. In general, the phi coefficients and consistency estimates within each condition showed both a small serial position effect (beginning and ending pairs more associated than middle pairs) and a stronger distance effect (adjacent pairs of letters more associated than distant pairs).

Eight of the 10 phi coefficients for words were greater than the respective indices for meaning items (binomial $p<.055$ ), all 10 coefficients were greater for meaning than for compare items $(p<.001)$, and eight were greater for compare than for novel items $(p<.055)$. Nine of the consistency estimates for words were greater than respective estimates for meaning items (binomial $p<.011)$, all 10 were greater for meaning than for compare items $(p<.001)$, and 9 were greater for compare than for novel items $(p<.011)$. On this basis, we concluded that the conditions differed in bivariate association. As overall estimates of this association, mean phi coefficients of the conditions were: words, .56 ; meaning, .48; compare, .33; and novel, .30; and mean probability of identical outcomes were: words, .82; meaning, .76; compare, .67; and novel, .56.

The ordinal magnitudes of these indices for the three types of pseudowords (meaning, compare, and novel) correspond to the ordinal magnitudes of their perceptual accuracy, in such a way that greater perceptual accuracy is accompanied by greater perceptual dependence. The 
probability of dependence coefficients occurring in the same order as accuracy scores by chance alone is .167 , and the probability of both occurring in the predicted order by chance is .027 . This correspondence suggests that task demands affect pseudoword perception through the perceptual integration that is incidental to satisfying the purpose of the task. Thus the differences in pseudoword perceptibility appear to depend on differences in the organization of structural knowledge, rather than on differences in the strength of that knowledge. In particular, meaning appears to affect pseudoword perception indirectly by serving as the occasion for establishing memories that preserve the experience of relatively integrated perceptual processing.

If pseudoword perception is controlled by perceptual integration of earlier experiences, and word perception also depends on episodic representation, then differences in perceptual dependence between words and pseudowords should predict differences in perceptual accuracy. However, whereas the perceptual interdependence of letters in words is apparently greater than that in meaning pseudowords, the accuracy of word perception is not significantly different from the accuracy of meaning perception in Experiments 1, 2, or 3.

An additional difficulty is that there seems to be reason to expect that words should have been better perceived than meaning items if supported by episodic representation. The words employed were of moderately high frequency, suggesting the availability of a large number of episodic traces, whereas subjects encountered the meaning pseudowords only five times in their lives. Thus on grounds of both perceptual dependence and number of experiences available, the lack of word superiority over meaning pseudowords casts doubt on the analogy of word and pseudoword perception.

\section{EXPERIMENT 4}

This argument leaves out an important factor. In all the experiments above, perception of meaning pseudowords was supported by previous encounters with those stimuli in the same context. In contrast, the words presented in the perception test, although generally familiar, were novel in the experimental context. If perception integrates item components not only with each other but also with characteristics of the setting in which the experience occurs, then item information will not be available independent of contextual reinstatement. Greater integration predicts more extreme performance rather than better performance (Whittlesea, 1987). The greater the integration of the trace, the more assistance the trace brings to perception, because the mutual generation of integrated elements offers an alternate route to identification. However, because components of integral traces are not independently accessible, greater integration also means less probability of activating a trace if components of the original experience are not reinstated, resulting in a failure of facilitation. In consequence, the greater number of traces of words and their greater integration may be offset by the unfamiliarity of the words in their test context. Thus if word perception is based on the same processes as pseudoword perception, the greater integration of words should result in an enduring perceptual advantage relative to meaning pseudowords if the words are preexposed in the test context.

\section{Method}

Subjects. Thirty undergraduate students participated for course credit.

Procedure. The procedure of Experiment 4 was identical to that of Experiment 3, except that an additional exposure phase was included, in which the subjects were shown the words to be used in the perceptual test and were required to copy them on paper. The number and order of word presentations were yoked to those of the two pseudoword conditions. The order of the three training conditions (words, meaning, and compare) was counterbalanced between subjects.

\section{Results and Discussion}

The probabilities of identifying letters in the perceptual test were: words, .83; meaning, .74; compare, .64; novel, .57; and single letters, .48. Analysis of variance indicated a significant effect of test condition $[F(4,116)$ $=36.77, M S \mathrm{e}=.015, p<.001]$, and Newman-Keuls a posteriori analysis indicated that all paired comparisons were significantly different $(p<.01)$. Thus when words have been presented in the context of a test, they receive a perceptual advantage over meaning pseudowords that does not occur otherwise, and this advantage endures at least $24 \mathrm{~h}$.

All 10 phi coefficients for words were greater than respective indices for meaning items (binomial $p<.001$ ), 8 were greater for meaning than for compare items $(p<$ $.055)$, and 8 were greater for compare than for novel items $(p<.055)$. All 10 probabilities of consistent outcomes were greater for words than for meaning items, for meaning than for compare items, and for compare than for novel items (binomial $p<.001$ in each case). Again, we concluded that the conditions differed in bivariate association. The mean phi coefficients for the four types of string stimuli were: words, .73; meaning, .46; compare, .37; and novel, .29; and the mean probabilities of identical outcomes were: words, .92; meaning, .77; compare, .70; and novel, .64.

The ordinal magnitudes of these indices for all four conditions correspond very well to the ordinal magnitudes of perceptual accuracy scores. The probability of both accuracy and association scores occurring in the same order by chance alone is .042 , and the probability of both $o c-$ curring in the predicted order by chance alone is .002 . Thus when familiarity in context is held constant, perceptual dependence predicts perceptual accuracy of both pseudowords and words.

Perception of meaning items was again found to be independent of the probability of recalling their meanings [point-biserial $\left.r^{2}=.002, t(358)=.96\right]$. As before, the correlation was not limited by low variance of percep- 
tion scores $(S D=.18)$ or by floor or ceiling effects in recall (mean proportion recalled $=.53$ ). This result corroborates the conclusion drawn in Experiment 3 that meaning has little direct effect on perception of meaning items. This dissociation does not necessarily indicate that meaning identification and perception are supported by information retained in separate traces. As argued by Jacoby and Witherspoon (1982) regarding a similar dissociation (between recognition and perceptual identification), such effects may be due to a difference in the cues and tasks used to access memory rather than to access of different memories.

\section{GENERAL DISCUSSION}

The purpose of these experiments was to investigate whether information about general, context-independent attributes of items, such as knowledge of their meaning or structure, is retained in memory and affects perception independent of information about particular experiences of the items. The observed differences in pseudoword perceptibility could not be attributed to differences in structural regularity or frequency, because items were counterbalanced between conditions and presented equal numbers of times. We argue that knowledge of meanings is not directly involved in the perceptual advantage of meaning pseudowords, because the identification of items is independent of identification of their meanings. This dissociation also suggests that the meaning advantage is not due to the formation of stronger codes, because factors promoting trace strength, such as encoding effort, should also increase the associative strength between an item and its meaning. Finally, because the different encoding tasks cause reliable differences in the interdependence of components in perception, we argue that the subject's knowledge of the structure of individual items preserves an organization given by the purpose of the original experience. Because differences in this organization reflected differences in the tasks in which items were originally encountered, and because these differences predicted the subject's ability to identify items, we conclude that pseudoword perceptibility depends on memory for particular experiences, jointly determined by the structural properties of the item and the purpose of the task.

The advantage of letters presented in novel pseudowords over letters tested alone may seem to limit the generality of this conclusion, because the novel pseudowords have no preestablished code. It may seem, therefore, that the perception of novel items depends on general properties of experience, such as orthographic regularity; indeed, the regularity of pseudowords predicts their advantage over single letters (McClelland \& Johnston, 1977). However, the perceptibility of both old and novel letter strings has been shown to depend on their similarity to particular items that the subject had encountered earlier, rather than on their similarity to the typical structure of previous experience (Whittlesea, 1987). Thus the correlation between the regularity and perceptibility of pseudowords appears to reflect reliance of perception on multiple par- ticular experiences rather than on direct coding of information about stimulus regularity.

The perception of natural words appears to follow the same principles that control pseudoword perception. Experiments 3 and 4 demonstrated that the accuracy of word perception is dependent on context and is predicted by the interdependence of components. These findings suggest that words, like pseudowords, are represented in terms of particular experiences. The general perceptual advantage of words may be due to their great interdependence, resulting from exposure in tasks conducive to holistic processing, such as processing for meaning, and to the large number of contexts in which words have been encountered, which would lead to robust similarity of at least some prior experiences to most contexts in which words will normally be encountered.

It would be of interest to know whether the particular organizations of stimuli given by particular experiences are permanent. This issue is important in evaluating models of word perception that assume canonical representation of structural information, such as McClelland and Rumelhart's (1981) interactive activation model. If idiosyncratic representations decay into a standard format over time, then such models may be good approximations to the processes involved in the perception of natural words. However, if memory preserves information about particular experiences indefinitely, then word perception could be better understood through models emphasizing episodic information.

Salasoo et al. (1985) observed that a single presentation of a pseudoword resulted in a perceptual advantage relative to words not preexposed in the test context, but that after a year the advantage had disappeared, although words and old pseudowords retained their superiority over novel pseudowords. Salasoo et al. interpreted these findings to suggest that episodic detail deteriorates with time, leaving behind a context-free representation of the item. However, it is unclear whether the particular organization of the pseudowords had decayed into a standard format, or whether the processing a subject applies to an item a year later is sufficiently different to reduce its similarity to the pool of traces available to assist perception. The experiments of this paper demonstrate that differences in interdependence of stimulus components do not decay rapidly, enduring at least $24 \mathrm{~h}$. This is consistent with findings of other investigators that if contexts are sufficiently distinctive, the effects of a prior presentation on perception may last a week (Jacoby, 1983a), and possibly as long as a year (Kolers, 1976). Because natural circumstances for encountering words are rich in contextual variation and in repetition, we conclude that memory for particular experiences may ordinarily exercise a strong and enduring influence on perception.

\section{REFERENCES}

Baron, J., \& Thurston, I. (1973). An analysis of the word superiority effect. Cognitive Psychology, 4, 207-228.

Brooks, L. R. (1987). Decentralized control of categorization: The role of prior processing episodes. In U. Neisser, Concepts and conceptual 
development: Ecological and intellectual factors in categorization. Cambridge: Cambridge University Press.

Cohen, N. J., \& SQuire, L. R. (1980). Preserved learning and retention of pattern-analyzing skill in amnesia: Dissociation of knowing how and knowing that. Science, 210, 207-210.

EsTEs, W. K. (1975). The locus of inferential and perceptual processes in letter identification. Journal of Experimental Psychology: General, 104, 122-145.

FIENBERG, S. E. (1977). The analysis of cross-classified categorical data. Cambridge, MA: MIT Press.

Gibson, E. J., ShurclifF, A., \& Yonas, A. (1970). Utilization of spelling patterns by deaf and hearing subjects. In H. Levin \& J. P. Williams (Eds.), Basic studies in reading. New York: Basic Books.

JaCOBY, L. L. (1983a). Perceptual enhancement: Persistent effects of an experience. Journal of Experimental Psychology: Learning, Memory, \& Cognition, 9, 21-38.

JACOBY, L. L. (1983b). Remembering the data: Analyzing interactive processes in reading. Journal of Verbal Learning \& Verbal Behavior, $22,485-508$.

JACOBY, L. L., \& BROOKS, L. R. (1984). Nonanalytic cognition: Memory, perception, and concept formation. In G. H. Bower (Ed.), The psychology of learning and motivation (Vol. 18). New York: Academic Press.

JACOBY, L. L., \& DALLAS, M. (1981). On the relationship between autobiographical memory and perceptual leaming. Joumal of Experimental Psychology: General, 3, 306-340.

JACOBY, L. L., \& WITHERSPOON, D. (1982). Remembering without awareness. Canadian Journal of Psychology, 36, 300-324.

Kolers, P. A. (1976). Reading a year later. Journal of Experimental Psychology: Human Learning \& Memory, 2, 554-565.

Kolers, P. A., Palef, S. R., \& Stelmach, L. B. (1980). Graphemic analysis underlying literacy. Memory \& Cognition, 8, 322-328.

MASON, M. (1975). Reading abilities and letter search time: Effects of orthographic structure defined by single-letter positional frequency. Journal of Experimental Psychology: General, 104, 146-166.

MCClelland, J. L., \& Johnston, J. C. (1977). The role of familiar units in perception of words and nonwords. Perception \& Psychophysics, 22, 249-261.

McClelland, J. L., \& Rumelhart, D. E. (1981). An interactive activation model of the effect of context in perception. Part I: An account of basic findings. Psychological Review, 88, 375-407.

MorTon, J. (1979). Facilitation in word recognition: Experiments causing change in the logogen model. In P. A. Kolers, M. E. Wrolstad, \& H. Bouma (Eds.), Proceedings of the conference on the process ing of visible language. New York: Plenum Press.

REICHER, G. M. (1969). Perceptual recognition as a function of meaningfulness of stimulus material. Journal of Experimental Psychology, 81 274-280.

RUMELhaRT, D. E., \& SIPLE, P. (1974). The process of recognizing tachistoscopically presented words. Psychological Review, 81, 99-118.
Salasoo, A., Shiffrin, R. M., \& Feustal, T. C. (1985). Building permanent memory codes: Codification and repetition effects in word identification. Journal of Experimental Psychology: General, 114, 50-77.

SMITH, S. M. (1979). Remembering in and out of context. Journal of Experimental Psychology: Human Learning \& Memory, 5, 460-471.

Tulving, E. (1972). Episodic and semantic memory. In E. Tulving $\&$ W. Donaldson (Eds.), Organization of memory. New York: Academic Press.

Tulving, E. (1983). Elements of episodic memory. London: Oxford University Press.

Tulving, E., Schacter, D. L., \& Stark, H. A. (1982). Priming effects in word-fragment completion are independent of recognition memory. Journal of Experimental Psychology: Learning. Memory, \& Cognition, 8, 336-342.

WARREN, C., \& Morton, J. (1982). The effects of priming on picture recognition. British Journal of Psychology, 73, 117-129.

WhEelER, D. D. (1970). Processes in word recognition. Cognitive Psychology, 1, 59-85.

Whittlesea, B. W. A. (1987). Preservation of specific experiences in the representation of general knowledge. Journal of Experimental Psychology: Learning, Memory, \& Cognition, 13, 3-17.

\section{NOTE}

1. Conceivably, subjects could improve their identification scores by guessing misperceived target letters on the basis of perceiving the context and remembering whole items from the training phase. The Reicher (1969) control that eliminates deliberate deduction could not be applied because of the use of whole report in Experiments 3 and 4. However, the Reicher control eliminates guessing only when the subject has no information about the target letter. The word advantage itself indicates that the context influences perception of the target, and this influence appears to consist of something like automatic activation of hypotheses about the target's identity, based on word and context information, that interact with perceptual information about the target. Indeed, this is the basis of interactive models of word perception (e.g., McClelland \& Rumelhart, 1981). The present research is aimed at understanding the form of knowledge in which information about contexts and words is preserved, in order to understand how it may interact with target information. Although our methodology risks application of a deliberate, deductive strategy, we would like to suggest that if subjects had employed this strategy consistently, we would have observed at least some correlation between perceptual accuracy and meaning recall.

(Manuscript received November 17, 1986; revision accepted for publication May 26, 1987.) 